\title{
1 Substrate-Specific Effects of Natural Genetic Variation on Proteasome 2 Activity
}

3 Mahlon A. Collins, Randi R. Avery, and Frank W. Albert

4 Department of Genetics, Cell Biology, and Development

5 University of Minnesota

6 Minneapolis, MN, U.S.A.

7 Co-Corresponding Authors:

8 Mahlon A. Collins (mahlon@umn.edu)

$9 \quad$ Frank W. Albert (falbert@umn.edu) 


\section{Abstract}

The bulk of targeted cellular protein degradation is performed by the proteasome, a multi-subunit complex consisting of the 19S regulatory particle, which binds, unfolds, and translocates substrate proteins, and the $20 \mathrm{~S}$ core particle, which degrades them. Protein homeostasis requires precise, dynamic control of proteasome activity. To what extent genetic variation creates differences in proteasome activity is almost entirely unknown. Using the ubiquitin-independent degrons of the ornithine decarboxylase and Rpn4 proteins, we developed reporters that provide high-throughput, quantitative measurements of proteasome activity in vivo in genetically diverse cell populations. We used these reporters to characterize the genetic basis of variation in proteasome activity in the yeast Saccharomyces cerevisiae. We found that proteasome activity is a complex, polygenic trait, shaped by variation throughout the genome. Genetic influences on proteasome activity were predominantly substrate-specific, suggesting that they primarily affect the function or activity of the 19S regulatory particle. Our results demonstrate that individual genetic differences create heritable variation in proteasome activity and suggest that genetic effects on proteasomal protein degradation may be an important source of variation in cellular and organismal traits.

\section{Introduction}

Protein homeostasis requires continuous degradative protein turnover. The vast majority of targeted protein degradation occurs through the concerted actions of the ubiquitin system and the proteasome (collectively, the ubiquitin-proteasome system [UPS]). The ubiquitin system is a series of enzymes that mark proteins for degradation by covalent attachment of the small protein ubiquitin. The proteasome is a multi-subunit complex that binds and degrades ubiquitinated cellular proteins $^{1-5}$. UPS protein degradation influences virtually all aspects of cellular physiology ${ }^{2,3,6}$, necessitating precise, dynamic control of proteasome activity. Accordingly, a diverse array of regulatory mechanisms control proteasome activity by altering the abundance, function, or composition of the cell's complement of proteasomes ${ }^{7}$. Imbalances between proteasome activity and the proteolytic needs of the cell adversely impact cellular viability and are implicated in numerous human diseases, including metabolic, immune, and neurodegenerative disorders ${ }^{8-13}$.

We recently established individual genetic differences as an important source of variation in UPS protein degradation ${ }^{14}$. Quantitative trait locus (QTL) mapping revealed widespread genetic influences on the activity of the ubiquitin system. DNA variants in ubiquitin system genes cause altered processing, recognition, and ubiquitination of UPS substrates ${ }^{14}$. However, whether genetic 
influences on the activity of the proteasome are equally numerous is unknown.

The proteasome consists of two functionally distinct modules. The $19 \mathrm{~S}$ regulatory particle selects, unfolds, and translocates substrates, which are then processively degraded by the $20 \mathrm{~S}$ core particle. DNA sequence differences may differentially affect the $19 \mathrm{~S}$ regulatory particle and the $20 \mathrm{~S}$ core particle. Genetic influences on the $19 \mathrm{~S}$ regulatory particle could manifest as substratespecific changes in protein degradation. In contrast, genetic influences on $20 \mathrm{~S}$ core particle activity should alter turnover similarly across substrates. To date, however, there have been no efforts to characterize genetic influences on proteasome activity, in part, because of a lack of suitable methods. Comprehensively characterizing genetic influences on proteasome activity with high statistical power requires assays that can provide high-throughput, quantitative, in vivo measurements of proteasomal protein degradation. Several high-throughput reporter systems have been developed to measure UPS activity ${ }^{14-17}$. However, these systems are responsive to changes in both ubiquitin system and proteasome activity.

A handful of endogenous cellular proteins are proteasomally degraded without ubiquitination ${ }^{18}$. These proteins contain short peptide sequences, termed ubiquitin-independent degrons, that promote rapid proteasomal degradation ${ }^{19-23}$. Ubiquitin-independent degrons simultaneously function as proteasome recognition elements that engage the 19S regulatory particle and unstructured initiation regions for $20 \mathrm{~S}$ core particle degradation ${ }^{18,20,22-26}$. These peptides are transferrable; conjugating a ubiquitin-independent degron to a heterologous protein converts it to a short-lived, ubiquitin-independent proteasome substrate ${ }^{22-24,26,27}$. This property has been leveraged to create genetically encoded, high-throughput reporters of proteasome activity ${ }^{20,28}$. However, in genetically diverse populations, the output of these systems is confounded by differences in reporter expression caused by individual genetic differences between cells ${ }^{29-32}$.

Here, we used the ubiquitin-independent degrons from ornithine decarboxylase (ODC) ${ }^{19,20}$ and Rpn $4^{21-23}$ to build high-throughput reporters for measuring proteasome activity in genetically diverse cell populations. We used these reporters to perform statistically powerful QTL mapping of the genetics of proteasome activity. Our results reveal that proteasome activity is a polygenic trait shaped by variation throughout the genome. Proteasome activity QTLs were largely substratespecific, suggesting that genetic variation shapes proteasome activity primarily through effects on the 19S regulatory particle. Individual genetic differences are an important source of variation in proteasome activity that may contribute to the complex genetic basis of the many cellular and 
organismal traits influenced by proteasomal protein degradation.

\section{Results}

\section{Single-Cell Measurements Reveal Genetic Differences in Proteasome Activity}

We sought to develop a reporter system capable of measuring proteasome activity with high throughput and quantitative precision without confounding from genetic influences on reporter expression. To do so, we built a series of tandem fluorescent timers (TFTs), fusions of two fluorescent proteins with distinct spectral profiles and maturation kinetics ${ }^{33,34}$. Our TFTs contained a faster-maturing green fluorescent protein (GFP) and a slower-maturing red fluorescent protein (RFP) (Figure 1A). The two fluorophores in the TFT mature at different rates. As a result, the RFP / GFP ratio changes over time. If the TFT's degradation rate is faster than the RFP's maturation rate, the negative $\log _{2}$ RFP / GFP ratio is directly proportional to the TFT's degradation rate (Figure 1B). The TFT's RFP / GFP ratio is independent of the reporter's expression level ${ }^{17}$, making it possible to use TFTs in genetically diverse cell populations without confounding from genetic influences on reporter expression ${ }^{14,17}$.

To relate the RFP / GFP ratio to proteasome activity, we fused the ubiquitin-independent degrons from the mouse ODC and yeast Rpn4 proteins to our TFTs. The ubiquitin-independent proteasomal degradation of mouse ODC is conserved in yeast and, unlike yeast ODC, does not require expression of antizyme for efficient proteasomal degradation ${ }^{20,24}$. We fused amino acids 410 through 461 of mouse ODC to the C-terminus of the sfGFP-mCherry TFT, consistent with the geometric requirements of the ODC degron ${ }^{20}$, to create the ODC TFT (Figure 1C). The Rpn4 protein contains a ubiquitin-independent degron in amino acids 1 to $80^{22,23}$. We fused this sequence to the N-terminus of the sfGFP-mCherry TFT to create the Rpn4 TFT (Figure 1C). We reasoned that the distinct recognition mechanisms, sequences, and geometric requirements of the ODC and Rpn4 degrons would allow us to distinguish substrate-specific genetic effects on the 19S regulatory particle from substrate-independent genetic effects on the 20S core particle.

We characterized the ODC and Rpn4 TFTs in single cells by flow cytometry. We first evaluated the sensitivity and dynamic range of each TFT by comparing the RFP / GFP ratio in the BY laboratory strain, which is closely related to the S288C reference strain, and a BY strain lacking the RPN4 gene (hereafter "BY rpn 4 "). RPN4 encodes a transcription factor for proteasome genes, and deleting $R P N_{4}$ reduces proteasome activity ${ }^{21,25,35}$. Deleting $R P N_{4}$ strongly reduced 
the negative $\log _{2}$ RFP / GFP output from the ODC and Rpn4 TFTs (t-test $p=1.4 \mathrm{e}-6$ and 1.6e-13, respectively; Figure 1D/E), showing that our TFTs provide sensitive measurements of proteasome activity. Consistent with previous reports ${ }^{18,26,27}$, in the BY strain the ODC TFT was more rapidly degraded than the Rpn4 TFT (t-test $p=6.9 \mathrm{e}-10$, Figure 1D/E). Taken together, our results show that our TFTs provide quantitative, substrate-specific readouts of proteasome activity.

To understand how natural genetic variation affects proteasome activity, we measured the output of the ODC and Rpn4 TFTs in two genetically divergent $S$. cerevisiae strains, BY and RM, a genetically divergent vineyard strain. The RM strain showed higher proteasome activity towards the ODC and Rpn4 TFTs than BY (t-test $p=1.9 \mathrm{e}-4$ and $1.2 \mathrm{e}-8$, respectively; Figure 1D/E). We observed a significant interaction between strain background and proteasome substrate such that the magnitude of the BY/RM strain difference was greater for the Rpn4 TFT than the ODC TFT (two-way ANOVA interaction $p=0.013$ ). Together, these results show that individual genetic differences create heritable, substrate-dependent variation in proteasome activity. 

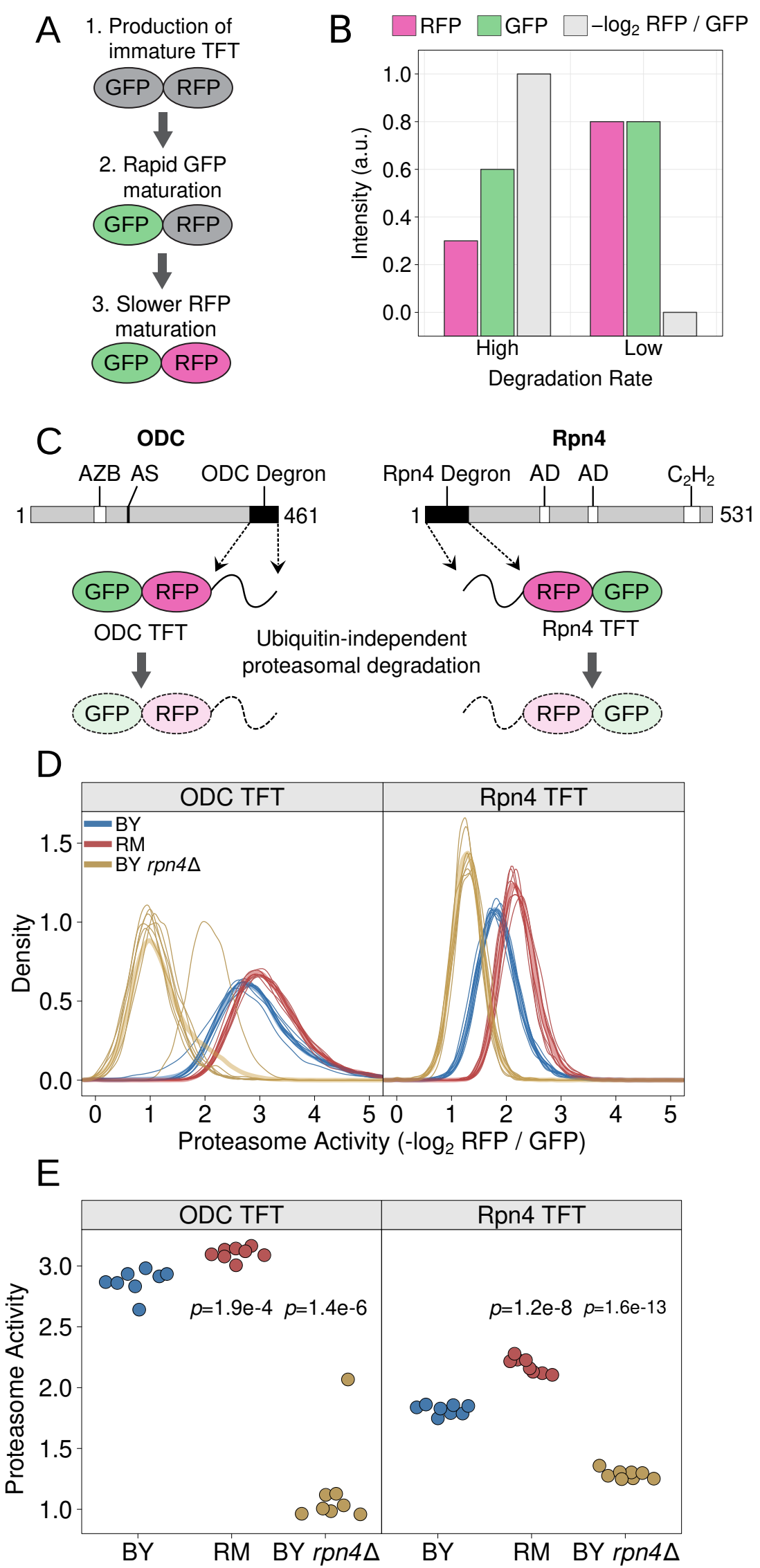
Figure 1: Design and characterization of proteasome activity reporters. A. Schematic of the production and maturation of a TFT. B. A bar plot created with hypothetical data shows how differences in a TFT's degradation rate influence the reporter's RFP, GFP, and negative $\log _{2}$ RFP / GFP levels. C. Diagram of mouse ODC and yeast Rpn4 showing the location of each protein's ubiquitin-independent degron. "AZB" = antizyme binding site, " $A S$ " = active site, " $A D$ " = transcriptional activation domain, " $\mathrm{C}_{2} \mathrm{H}_{2}$ " $=\mathrm{C}_{2} \mathrm{H}_{2}$ zinc finger DNA binding domain. D. Density plots of the negative $\log _{2}$ RFP / GFP ratio from 10,000 cells for each of 8 independent biological replicates per strain per reporter for the indicated strains and TFTs. Thin, opaque lines show individual biological replicates and thicker, transparent lines show the group average for the indicated strains. E. The median from each biological replicate in $D$. is plotted as a stripchart. t-test p-values are shown for the indicated strain versus $B Y$.

\section{Bulk Segregant Analysis Identifies Complex, Polygenic Influences on Proteasome Activity}

To map genetic influences on proteasome activity, we used our ODC and Rpn4 TFTs to perform bulk segregant analysis, a statistically powerful genetic mapping method that compares large numbers of individuals with extreme values for a trait of interest selected from a genetically diverse population ${ }^{14,32,36-38}$. In our implementation, the method identifies proteasome activity QTLs, regions of the genome containing one or more DNA variants influencing proteasome activity. We created genetically diverse cell populations by mating BY strains harboring either the ODC or Rpn4 TFT with RM and sporulating the resulting diploids (Figure 2A). We collected cells from the $2 \%$ tails of the proteasome activity distribution using fluorescence-activated cell sorting (FACS) (Figure 2B-E) and used whole-genome sequencing to determine the allele frequency difference between the high and low UPS activity pools at each DNA variant. At QTLs affecting proteasome activity, the allele frequencies will be significantly different between pools, while at unlinked loci the allele frequencies will be the same. We called significant QTLs using a logarithm of the odds (LOD) threshold previously determined to produce a $0.5 \%$ false discovery rate for TFT-based genetic mapping ${ }^{14}$ (see "Methods") and retained only QTLs detected at genome-wide significance in both of two independent biological replicates. To visualize the direction and magnitude of QTL effect sizes, we plotted the allele frequency difference along each chromosome (Supplementary Figures 1 and 2). We identified 11 QTLs for the ODC TFT and 7 QTLs for the Rpn4 TFT (Figure 3, Table 1). The distribution of proteasome activity QTL effect sizes, as measured by the allele 
A

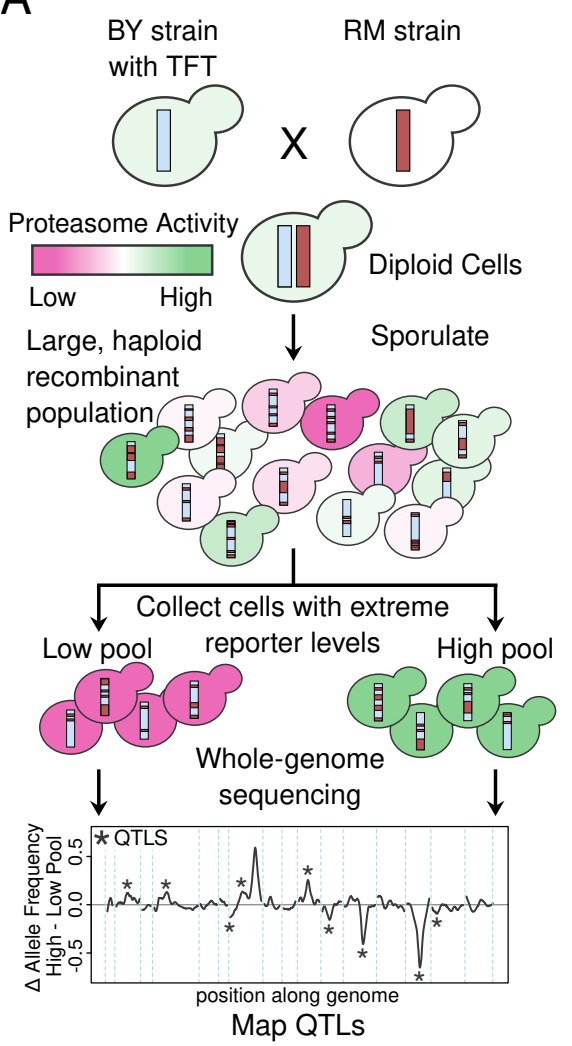

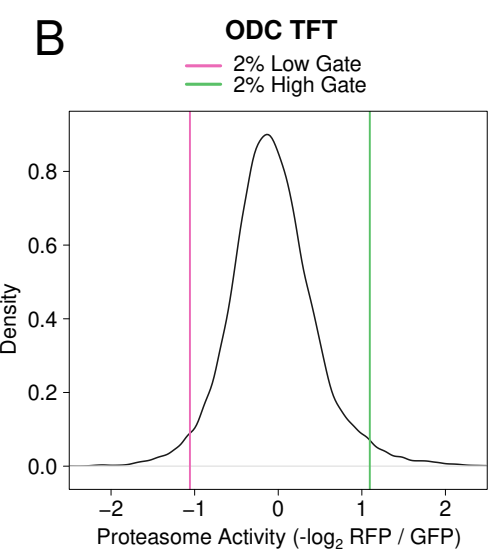

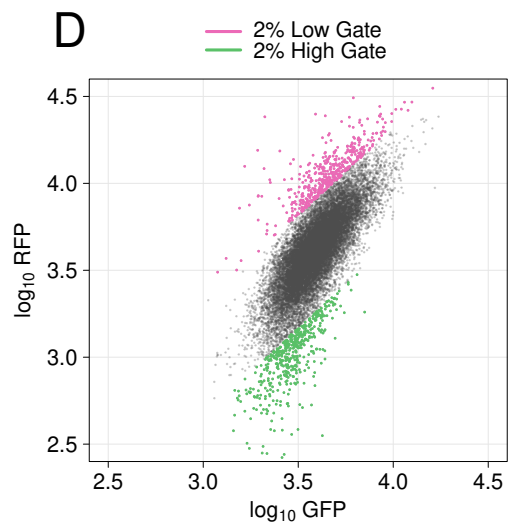

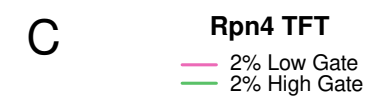
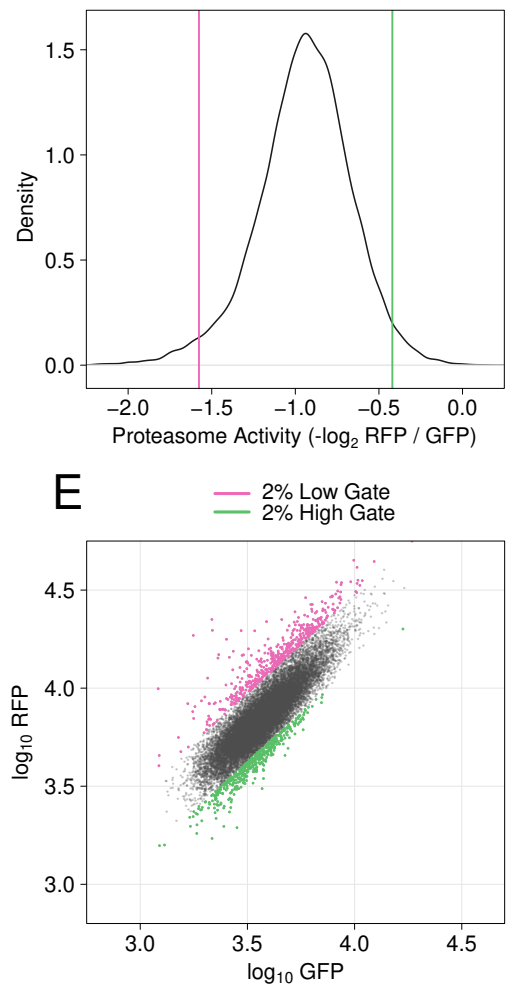

Figure 2: Mapping genetic influences on proteasome activity using bulk segregant analysis. A. Schematic of the experimental approach. B./C. Proteasome activity distributions for the ODC TFT (B.) and Rpn 4 TFT (C.). Vertical lines show the gates used to collect cells with extreme high or low proteasome activity. D./E. Backplot of cells collected using the gates in B./C. onto a scatter plot of GFP and RFP for the ODC (D.) and Rpn4 (E.) TFTs. 


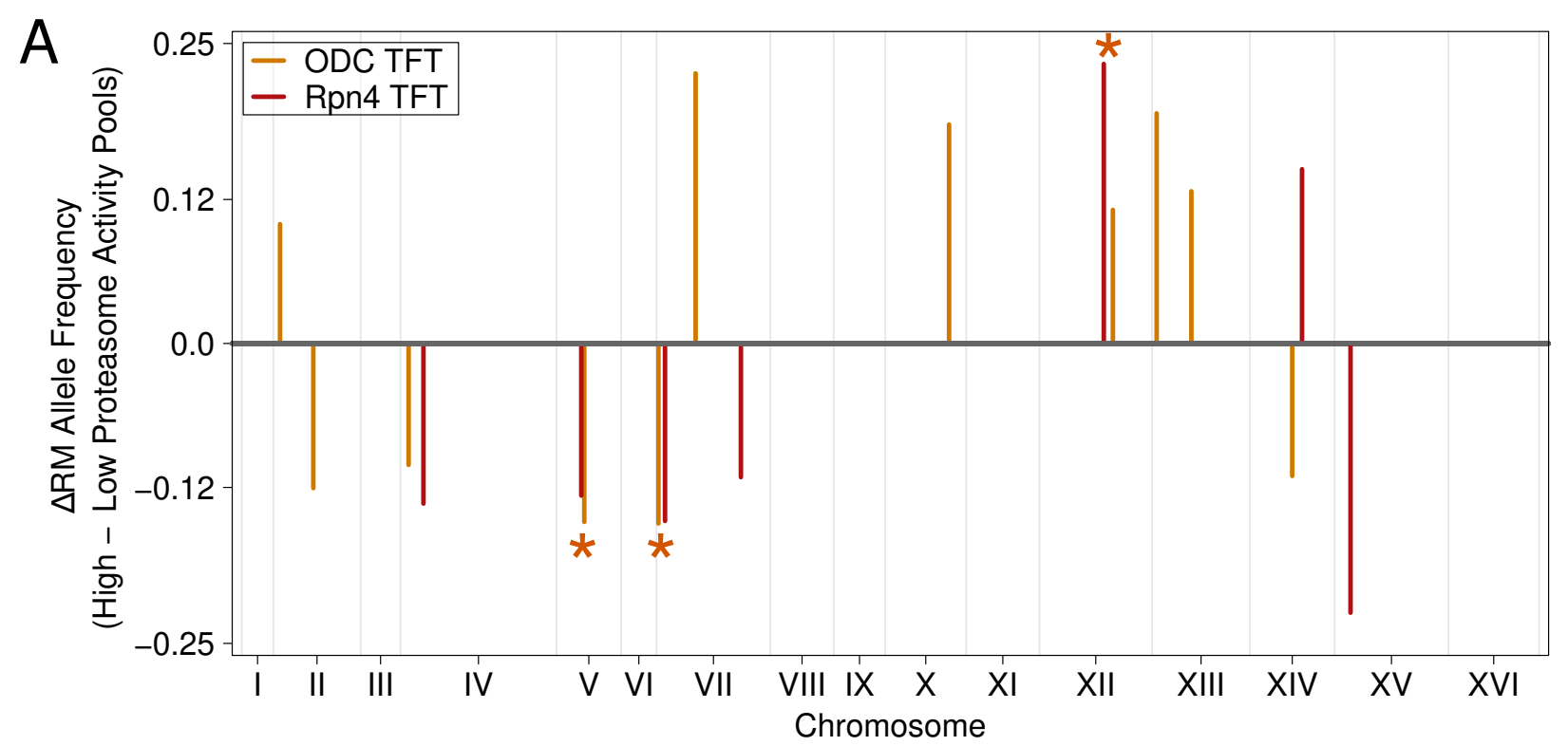

B

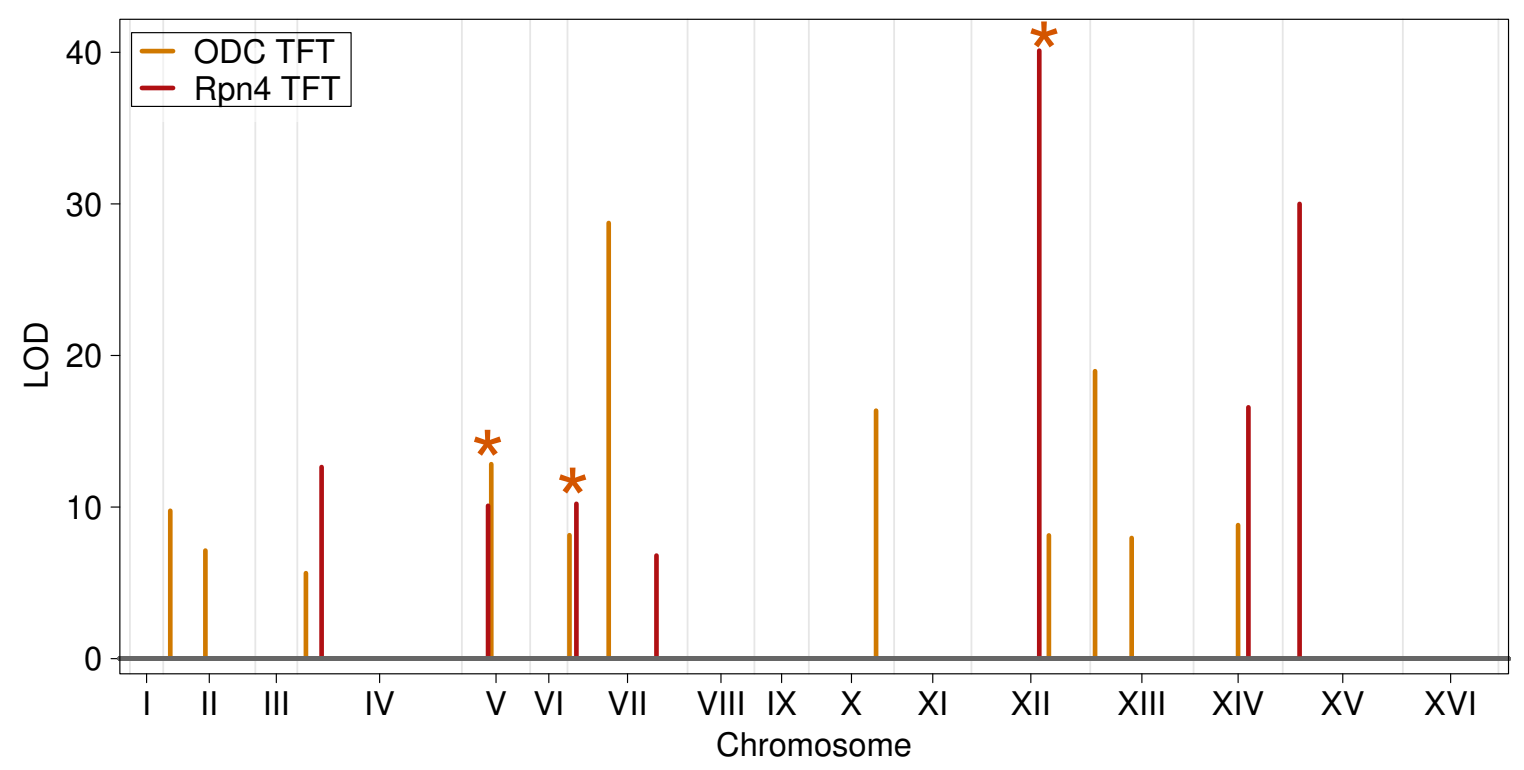

Figure 3: Proteasome activity QTLs for the ODC and Rpn4 TFTs. A. Line plot of the RM allele frequency difference between the high and low UPS activity pools at the peak position for statistically significant proteasome activity QTLs. B. As in A., but for the LOD score for proteasome activity QTLs. In A./B. asterisks denote shared QTLs detected with the ODC and Rpn4 TFTs. 


\begin{tabular}{lcccccc}
\hline Reporter & Chromosome & LOD & AFD & Peak Position & Left Index & Right Index \\
\hline ODC TFT & IIa & 9.76 & 0.099 & 69800 & 32850 & 107100 \\
ODC TFT & IIb & 7.13 & -0.121 & 418100 & 358850 & 462650 \\
ODC TFT & IVa & 5.64 & -0.101 & 85150 & 30400 & 127400 \\
ODC TFT & V & 12.83 & -0.149 & 291350 & 247700 & 325650 \\
ODC TFT & VIIa & 8.14 & -0.150 & 20000 & 0 & 52800 \\
ODC TFT & VIIb & 28.74 & 0.225 & 409000 & 390050 & 431700 \\
ODC TFT & X & 16.36 & 0.182 & 666850 & 649350 & 691550 \\
ODC TFT & XII & 8.13 & 0.111 & 768150 & 666200 & 846700 \\
ODC TFT & XIIIa & 18.96 & 0.192 & 47800 & 25200 & 75850 \\
ODC TFT & XIIIb & 7.96 & 0.127 & 410900 & 377350 & 450100 \\
ODC TFT & XIVa & 8.81 & -0.110 & 441750 & 381400 & 501600 \\
Rpn4 TFT & IVb & 12.64 & -0.133 & 240600 & 213200 & 309150 \\
Rpn4 TFT & V & 10.09 & -0.127 & 259650 & 218250 & 294900 \\
Rpn4 TFT & VIIa & 10.21 & -0.148 & 88550 & 53550 & 141350 \\
Rpn4 TFT & VIIc & 6.80 & -0.111 & 882500 & 840650 & 926150 \\
Rpn4 TFT & XII & 40.11 & 0.233 & 672850 & 661800 & 685750 \\
Rpn4 TFT & XIVb & 16.58 & 0.145 & 544150 & 497300 & 574600 \\
Rpn4 TFT & XV & 30.00 & -0.224 & 167400 & 142600 & 186200 \\
\hline
\end{tabular}

Table 1: Proteasome activity QTLs. The table lists all QTLs detected, sorted first by reporter, then by chromosome. Lowercase letters following chromosome numbers are used to distinguish QTLs on the same chromosome. "LOD", logarithm of the odds; "AFD", RM allele frequency difference (high proteasome activity pool minus low proteasome activity pool) at the QTL peak position. Each number is the average value calculated from two independent biological replicates for a given QTL. "Peak Position", "Left Index", and "Right Index" refer to base pair positions on the indicated chromosome.

\section{Genetic Influences on Proteasome Activity are Predominantly Substrate-Specific}

We evaluated the extent to which QTLs detected with the ODC and Rpn4 TFTs overlapped, reasoning that shared QTLs may reflect substrate-independent genetic influences on proteasome activity, such as altered 20S core particle activity. Only 3 of 18 QTLs (on chromosomes V, VII, and XII; Figure 3, Table 1) were shared between the ODC and Rpn4 TFTs. Because QTLs 
that influence the $20 \mathrm{~S}$ core particle would be expected to affect both TFTs, the high degree of observed QTL specificity between reporters suggests that variant effects on proteasome activity predominantly influence substrate selection by the 19S regulatory particle.

We recently mapped genetic effects on the UPS N-end Rule pathway ${ }^{14}$, in which ubiquitinated N-terminal amino acids function as $19 \mathrm{~S}$ regulatory particle recognition elements ${ }^{6}$. We compared the overlap of the 149 UPS N-end rule activity QTLs (obtained earlier using 20 N-end rule reporters ${ }^{14}$ ) and the 18 proteasome activity QTLs to better understand the substrate specificity of genetic influences on proteasome activity. Six proteasome activity QTLs did not overlap any of the 149 UPS activity QTLs (Figure 4, Supplementary Table 1). Even though the ODC/Rpn4 degrons and poly-ubiquitin chains each act as proteasome binding tags, these substrate-specific QTLs suggest that genetic variation may differentially alter the processing, function, or recognition of these elements. The remaining 12 proteasome activity QTLs overlapped with varying numbers of the 20 UPS N-end rule reporters, with a range of 1 to 10 and a median of 3 (Figure 4, Supplementary Table 1). The low extent of overlap in proteasome activity QTLs and UPS N-End Rule QTLs further highlights the high degree of substrate specificity in genetic influences on proteasome activity. 


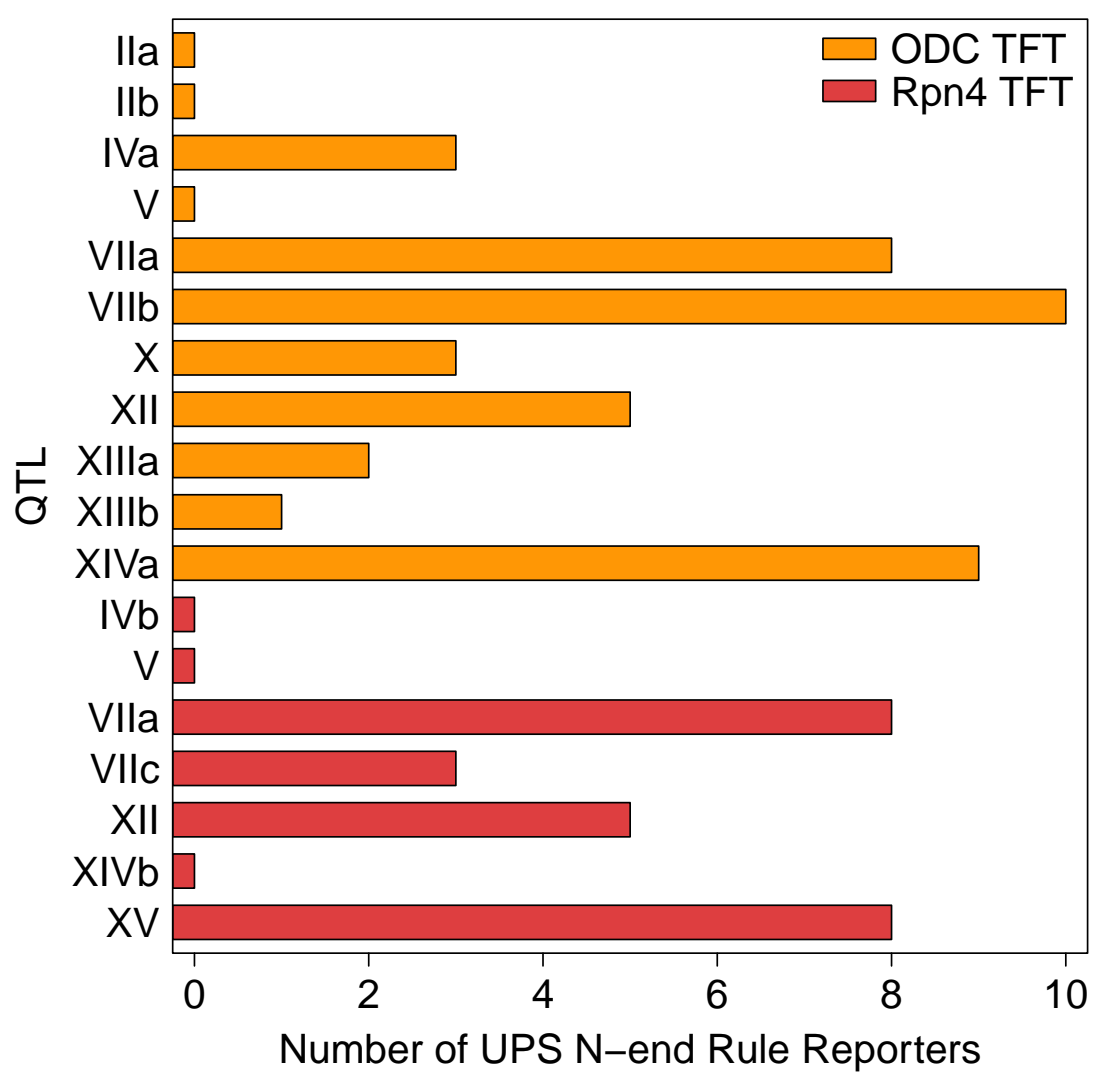

Figure 4: Overlap of proteasome activity QTLs with UPS N-end Rule QTLs. The barplot shows the number of UPS N-end Rule reporters (of a possible 20) for which a given proteasome activity QTL was detected. QTLs on the $y$-axis are labeled as in Table 1.

We previously used QTLs detected with multiple UPS N-end rule reporters to nominate and validate candidate causal QTL genes ${ }^{14}$. We applied this approach to our proteasome activity QTLs to gain insights into the mechanisms by which genetic variation may shape proteasome activity. A QTL on chromosome XII was detected with the ODC and Rpn4 TFTs and 5 N-end Rule reporters (Figure 4, Supplementary Table 1). The QTL's median peak position at base pair 665,600 occurs in the immediate vicinity of $H A P 1$, a transcription factor at which BY carries a transposon insertion that influences a variety of traits in this cross of yeast strains ${ }^{31,39,40}$. A QTL on chromosome XIV was detected with the ODC TFT and 9 UPS N-end rule reporters and its peak at base pair 464,275 was located near the MKT1 gene (Figure 4, Supplementary Table 1). MKT1 encodes an RNA-binding protein that also influences numerous traits in the BY/RM $\operatorname{cross}^{31,31,32,37,41}$. These QTLs thus likely shape proteasome activity through indirect mechanisms. The chromosome VIIb QTL was detected using the ODC TFT and 10 UPS N-end rule TFTs (Figure 4, Supplementary Table 1). 
The median peak position of this QTL (at base pair 409,450) occurs in the immediate vicinity of RPT6, an ATPase of the 19S regulatory particle. The RM allele of this QTL increases proteasome or UPS activity for all reporters for which the QTL was detected. RPT6 expression is increased by the RM allele at this locus ${ }^{31}$, making RPT6 a strong candidate causal gene for this QTL. This region was not linked to the output of the Rpn4 TFT, suggesting that the effect of variants at RPT6 are likely to affect the 19S regulatory particle in a substrate-specific manner.

\section{Discussion}

Protein homeostasis depends on precise, dynamic control of proteasome activity. However, the factors that create physiological and pathological variation in proteasome activity remain incompletely understood. Using a statistically powerful genetic mapping method, we have established individual genetic differences as an important source of heritable variation in proteasome activity. Genetic influences on proteasome activity are numerous and, similar to other polygenic traits $^{40,42,43}$, comprise a continuous distribution of effect sizes, with many QTLs of small effect and few QTLs of large effect. Our results add to the emerging picture of the complex effects of genetic variation on the UPS, which include widespread effects on the activity of both the ubiquitin system ${ }^{14}$ and, as we show here, the proteasome.

The proteasome consists of two functionally distinct modules. The 19S regulatory particle binds, unfolds, and translocates proteins to the $20 \mathrm{~S}$ core particle, which processively degrades them ${ }^{2-5}$. By mapping genetic influences on two ubiquitin-independent proteasome substrates with distinct sequences, geometries, and recognition mechanisms ${ }^{18-23,44}$, we observed that genetic influences on proteasome activity were primarily substrate-specific (Figure 3, Table 1). Because all proteasome substrates are processed by the $20 \mathrm{~S}$ core particle, DNA variants that influence the $20 \mathrm{~S}$ core particle would be expected to affect all substrates, even if some effects may be missed due to incomplete power to detect QTLs. In contrast, the high degree of substrate-specificity we observed suggests that genetic influences on the $19 \mathrm{~S}$ regulatory particle are more numerous than those affecting the 20S core particle. This notion is consistent with the multiple functions served by the 19S core particle. DNA sequence differences may influence the 19S regulatory particle's substrate binding, unfolding, or translocation to the $20 \mathrm{~S}$ core particle ${ }^{2,4}$. The $20 \mathrm{~S}$ core particle is highly evolutionarily conserved and, unlike the eukaryote-specific $19 \mathrm{~S}$ regulatory particle, is found in eukaryotes, bacteria, and archaea ${ }^{45,46}$. Thus, variation in $20 \mathrm{~S}$ core particle genes or in genes with trans-acting influences on $20 \mathrm{~S}$ core particle function or activity may be rapidly purged by purifying 
selection.

The numerous proteasome activity QTLs we have identified add new insight into how genetic variation shapes the molecular effectors of cellular protein degradation. We recently mapped the genetics of the UPS N-end rule pathway and discovered multiple DNA variants that alter the activity of several components of the ubiquitin system ${ }^{14}$. Here, we show that genetic effects on proteasome activity are similarly numerous. However, our analysis of QTL sharing suggests relatively little overlap in the sources of heritable variation in the ubiquitin system and the proteasome (Figure 4). Although many stimuli, such as protein misfolding or heat shock, cause similar changes in the activity of the ubiquitin system and the proteasome, recent work shows that these systems can also be regulated independently and function autonomously of one another ${ }^{7,47}$. For example, ubiquitination can initiate events besides proteasomal protein degradation, including lysosomal protein degradation, altered protein subcellular localization, and signaling cascade activation $^{47-49}$. Likewise, a number of cellular proteins are bound and degraded by the proteasome without modification by the ubiquitin system ${ }^{18}$. Thus, predicting how genetic variation shapes the degradation of UPS substrates will require consideration of genetic effects on both the ubiquitin system and the proteasome.

We have developed a reporter system that provides high-throughput, quantitative, in vivo measurements of proteasome activity in genetically diverse cell populations. The elements in our reporters function in many other eukaryotic organisms ${ }^{17,22,24}$ and applying this system to other species may clarify how genetic effects on proteasome activity shape cellular and organismal traits. Alleles of proteasome genes confer beneficial traits in plants, including heat tolerance ${ }^{50}$ and resistance to multiple pathogens ${ }^{51,52}$. Mutations in proteasome genes have been linked to a strikingly diverse array of human diseases, including coronary artery disease $\mathrm{e}^{53}$, ischaemic stroke $\mathrm{e}^{54}$, intellectual disability $^{55,56}$, deafness ${ }^{57}$, morphological abnormalities ${ }^{57}$, and Graves' disease ${ }^{58,59}$. However, the vast majority of studies linking variation in proteasome genes to organismal phenotypes have not tested whether and how the identified mutations alter proteasome activity. Applying our reporter system to proteasome gene variants would provide insight into the mechanisms and magnitude of the effects of these variants. Heritable variation in traits regulated by the proteasome may also result from the cumulative contributions of many alleles with small effects. Applying the mapping strategy developed here or using our reporters with other recently-developed mapping approaches ${ }^{60}$ may provide new insights into the genetic basis of a variety of cellular and organismal traits, including the many diseases marked by aberrant proteasome activity. 


\section{Materials and Methods}

\section{Tandem Fluorescent Timer (TFT) Reporters of Proteasome Activity}

We used TFTs, fusions of two fluorescent proteins with distinct spectral profiles and maturation kinetics, to measure proteasome activity. The most common TFT implementation consists of a faster-maturing green fluorescent protein (GFP) and a slower-maturing red fluorescent protein $(\mathrm{RFP})^{17,33,34,61}$. Because the two fluorescent proteins mature at different rates, the RFP / GFP ratio changes over time. If the TFT's degradation rate is faster than the RFP's maturation rate, the negative $\log _{2}$ RFP / GFP ratio is directly proportional to the TFT's degradation rate ${ }^{17,33}$. The RFP / GFP ratio is also independent of the TFT's expression level, ${ }^{17,33}$, enabling high-throughput, quantitative measurements of TFT turnover in genetically diverse cell populations ${ }^{14,17}$. All TFTs in the present study contained superfolder GFP (sfGFP) ${ }^{62}$ and mCherry ${ }^{63}$ separated by an unstructured 35 amino acid peptide sequence to minimize fluorescence resonance energy transfer ${ }^{17}$.

To measure proteasome activity with our TFTs, we fused the ubiquitin-independent degrons from the mouse ornithine decarboxylase (ODC) and yeast Rpn4 proteins to our sfGFP-mCherry TFTs. Ornithine decarboxylase (an enzyme in polyamine biosynthesis) contains a ubiquitin-independent degron in its C-terminal 37 amino acids ${ }^{19,20,27,44}$. Rpn4 (a transcription factor for proteasome genes) contains a ubiquitin-independent degron in its N-terminal 80 amino acids ${ }^{21,22,25}$. Both degrons are recognized and bound by the 19S regulatory particle without ubiquitin conjugation and function as unstructured initiation regions ${ }^{64}$ for $20 \mathrm{~S}$ core particle degradation. Attaching either degron to a heterologous protein converts it into a short-lived proteasomal substrate with half-lives of approximately 5 minutes for the ODC degron and 20 minutes for the Rpn 4 degron ${ }^{18,26,27}$. The ODC and Rpn4 degron sfGFP-mCherry TFTs thus provide direct, quantitative, substrate-specific readouts of proteasome activity (Figure 1).

We used a previously described approach ${ }^{14}$ to construct TFT reporters and yeast strains harboring TFTs. Each TFT contained the constitutively active TDH3 promoter, the ADH1 terminator, sfGFP, mCherry, and the Kanmx selection module ${ }^{65}$. TFTs were constructed so that the ubiquitinindependent degron was immediately adjacent to mCherry (Figure 1C), consistent with established guidelines for optimizing TFT function ${ }^{34}$. We used BFA0190 as the plasmid backbone for all TFT plasmids. BFA0190 contains $734 \mathrm{bp}$ of sequence upstream and $380 \mathrm{bp}$ of sequence downstream of the LYP1 ORF separated by a SwaI restriction site. We inserted TFT reporters into BFA0190 by digesting the plasmid with SwaI and inserting TFT components between the LYP1 flanking 
sequences using isothermal assembly cloning (Hifi Assembly Cloning Kit; New England Biolabs [NEB], Ipswich, MA, USA). The 5' and 3' LYP1 flanking sequences in each TFT plasmid contain natural SacI and BglII restriction sites, respectively. We produced linear DNA transformation fragments by digesting TFT-containing plasmids with SacI and BgIII and gel purifying the fragments (Monarch Gel Purification, NEB). Genomic integration of each linear transformation fragment results in deletion of the $L Y P 1$ gene, allowing selection for TFT integration at the $L Y P 1$ locus using the toxic amino acid analogue thialysine (S-(2-aminoethyl)-L-cysteine hydrochloride) $)^{66-68}$ and G418

\section{Yeast Strains and Handling}

\section{Yeast Strains}

We used two genetically divergent Saccharomyces cerevisiae yeast strains for characterizing our proteasome activity TFTs and mapping genetic influences on proteasome activity. The haploid BY strain (genotype: MATa his3 $\Delta$ ho $\Delta$ ) is a laboratory strain that is closely related to the $S$. cerevisiae S288C reference strain. The haploid RM strain is a vineyard isolate with genotype MAT $\alpha$ can1 $\Delta::$ STE2pr-SpHIS5 his3 $\Delta::$ NatMX AMN1-BY ho $\Delta:: H p h M X$ URA3-FY. BY and RM differ, on average, at 1 nucleotide per 200 base pairs, such that approximately 45,000 single nucleotide variants (SNVs) between the strains can serve as markers in a genetic mapping experiment ${ }^{32,36,37,40}$. We also engineered a BY strain lacking the $R P N 4$ gene (hereafter "BY rpn4 $\Delta$ ") to characterize the sensitivity and dynamic range of our TFT reporters. We replaced the RPN4 gene with the NatMX cassette, which confers resistance to the antibiotic nourseothricin ${ }^{65}$. To do so, we transformed BY with a DNA fragment created by PCR amplifying the NatMX cassette from plasmid from Addgene plasmid \#35121 (a gift from John McCusker) using primers with 40 bp of homology to the 5' upstream and 3' downstream sequences of RPN4 using the transformation procedure described below. Strain genotypes are presented in Table 2.

\begin{tabular}{|c|c|c|c|}
\hline Short Name & Genotype & Antibiotic Resistance & Auxotrophies \\
\hline BY & MATa his3 $\mathrm{h}$ ho & & histidine \\
\hline \multirow[t]{2}{*}{$\mathrm{RM}$} & 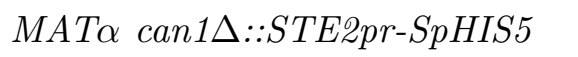 & clonNAT, hygromycin & histidine \\
\hline & 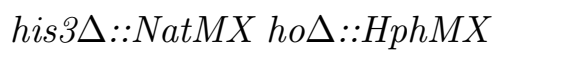 & & \\
\hline $\mathrm{BY} \operatorname{rpn} 4 \Delta$ & 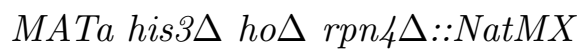 & clonNAT & histidine \\
\hline
\end{tabular}

Table 2: Strain genotypes 
The media formulations for all experiments are listed in Table 3. Synthetic complete media powders (SC -lys and SC -his -lys -ura) were obtained from Sunrise Science (Knoxville, TN, USA). We added the following reagents at the following concentrations to yeast media where indicated: G418, $200 \mathrm{mg} / \mathrm{mL}$ (Fisher Scientific, Pittsburgh, PA, USA); ClonNAT (nourseothricin sulfate, Fisher Scientific), $50 \mathrm{mg} / \mathrm{L}$; thialysine (S-(2-aminoethyl)-L-cysteine hydrochloride; MilliporeSigma, St. Louis, MO, USA), $50 \mathrm{mg} / \mathrm{L}$; canavanine (L-canavanine sulfate, MilliporeSigma), $50 \mathrm{mg} / \mathrm{L}$.

\begin{tabular}{lll}
\hline Media Name & Abbreviation & Formulation \\
\hline Yeast-Peptone-Dextrose & YPD & $10 \mathrm{~g} / \mathrm{L}$ yeast extract \\
& & $20 \mathrm{~g} / \mathrm{L}$ peptone \\
& & $20 \mathrm{~g} / \mathrm{L}$ dextrose \\
\hline Synthetic Complete & SC & $6.7 \mathrm{~g} / \mathrm{L}$ yeast nitrogen base \\
& & $1.96 \mathrm{~g} / \mathrm{L}$ amino acid mix -lys \\
& $20 \mathrm{~g} / \mathrm{L}$ dextrose \\
\hline Haploid Selection & SGA & $6.7 \mathrm{~g} / \mathrm{L}$ yeast nitrogen base \\
& & $1.74 \mathrm{~g} / \mathrm{L}$ amino acid mix -his -lys -ura \\
& & $20 \mathrm{~g} / \mathrm{L}$ dextrose \\
\hline Sporulation & $1 \mathrm{~g} / \mathrm{L}$ yeast extract \\
& SPO & $10 \mathrm{~g} / \mathrm{L}$ potassium acetate \\
& & $0.5 \mathrm{~g} / \mathrm{L}$ dextrose \\
\hline
\end{tabular}

Table 3: Media Formulations

\section{Yeast Transformations}

We used the lithium acetate/single-stranded carrier DNA/polyethyline glycol (PEG) method for all yeast transformations ${ }^{69}$. In brief, yeast strains were inoculated into $5 \mathrm{~mL}$ of YPD liquid medium for overnight growth at $30^{\circ} \mathrm{C}$. The next day, we diluted $1 \mathrm{~mL}$ of each saturated culture into 50 $\mathrm{mL}$ of fresh YPD and grew cells for 4 hours. Cells were washed in sterile ultrapure water and then in transformation solution 1 (10 mM Tris $\mathrm{HCl}$ [pH 8.0], $1 \mathrm{mM}$ EDTA [pH 8.0], and $0.1 \mathrm{M}$ lithium acetate). After each wash, we pelleted the cells by centrifugation at 3,000 rpm for 2 minutes in a benchtop centrifuge and discarded supernatants. After washing, cells were suspended in $100 \mu \mathrm{L}$ of transformation solution 1 along with $50 \mu \mathrm{g}$ of salmon sperm carrier DNA and $300 \mathrm{ng}$ of transforming DNA and incubated at $30^{\circ} \mathrm{C}$ for 30 minutes with rolling. Subsequently, $700 \mu \mathrm{L}$ of transformation 
solution 2 (10 mM Tris $\mathrm{HCl}$ [pH 8.0], $1 \mathrm{mM}$ EDTA [pH 8.0], and 0.1 M lithium acetate in 40\% PEG) was added to each tube, followed by a 30 minute heat shock at $42{ }^{\circ} \mathrm{C}$. Transformed cells were then washed in sterile, ultrapure water, followed by addition of $1 \mathrm{~mL}$ of liquid YPD medium to each tube. Cells were incubated in YPD for 90 minutes with rolling at $30^{\circ} \mathrm{C}$ to allow for expression of antibiotic resistance cassettes. We then washed the cells with sterile, ultrapure water and plated $200 \mu \mathrm{L}$ of cells on solid SC -lys medium with G418 and thialysine, and, for strains with the NatMX cassette, clonNAT. We single-colony purified multiple independent colonies (biological replicates) from each transformation plate for further analysis as indicated in the text. Reporter integration at the targeted genomic locus was verified by colony $\mathrm{PCR}^{70}$.

\section{Yeast Mating and Segregant Populations}

We used a modified synthetic genetic array (SGA) methodology ${ }^{67,68}$ to create populations of genetically variable, recombinant cells ("segregants") for genetic mapping. BY strains with either ODC or Rpn4 TFTs were mixed with the RM strain on solid YPD medium and grown overnight at $30^{\circ} \mathrm{C}$. We selected for diploid cells (successful BY/RM matings) by streaking mixed BY/RM cells onto solid YPD medium containing G418, which selects for the KanMX cassette in the TFT in the BY strain, and clonNAT, which selects for the NatMX cassette in the RM strain). Diploid cells were inoculated into $5 \mathrm{ml}$ of liquid YPD and grown overnight at $30^{\circ} \mathrm{C}$. The next day, cultures were washed with sterile, ultrapure water, and resuspended in $5 \mathrm{~mL}$ of SPO liquid medium (Table 3). We induced sporulation by incubating cells in SPO medium at room temperature with rolling for 9 days. After confirming sporulation by brightfield microscopy, we pelleted $2 \mathrm{~mL}$ of cells, which were then washed with $1 \mathrm{~mL}$ of sterile, ultrapure water, and resuspended in $300 \mu \mathrm{L}$ of $1 \mathrm{M}$ sorbitol containing 3 U of Zymolyase lytic enzyme (United States Biological, Salem, MA, USA) to degrade ascal walls. Asci were digested for 2 hours at $30{ }^{\circ} \mathrm{C}$ with rolling. Spores were then washed with 1 $\mathrm{mL}$ of $1 \mathrm{M}$ sorbitol, vortexed for 1 minute at the highest intensity setting, and resuspended in sterile ultrapure water. We confirmed the release of cells from asci by brightfield microscopy and plated $300 \mu \mathrm{l}$ of cells onto solid SGA medium containing G418 and canavanine. This media formulation selects for haploid cells with (1) a TFT via G418, (2) the MATa mating type via the Schizosaccharomyces pombe HIS5 gene under the control of the STE2 promoter (which is only active in MATa cells), and (3) replacement of the CAN1 gene with S. pombe HIS5 via the toxic arginine analog canavanine ${ }^{67,68}$. Haploid segregants were grown for 2 days at $30^{\circ} \mathrm{C}$ and harvested by adding $10 \mathrm{~mL}$ of sterile, ultrapure water and scraping the cells from each plate. Each segregant population cell suspension was centrifuged at $3000 \mathrm{rpm}$ for 10 minutes and resuspended in $1 \mathrm{~mL}$ of SGA medium. We added $450 \mu \mathrm{L}$ of $40 \%(\mathrm{v} / \mathrm{v})$ sterile glycerol solution to $750 \mu \mathrm{L}$ to each segregant culture and 
stored this mixture in screw cap cryovials at $-80^{\circ} \mathrm{C}$. We stored 2 independent sporulations each of the ODC and Rpn4 degron TFT-containing segregants (derived from our initial matings) as independent biological replicates.

\section{Flow Cytometry and Fluorescence-Activated Cell Sorting}

\section{Flow Cytometry}

We characterized our proteasome activity TFTs using flow cytometry. For all flow cytometry experiments, we inoculated yeast strains into $400 \mu \mathrm{L}$ of liquid SC -lys medium with G418 for overnight growth in $2 \mathrm{~mL} 96$ well plates at $30^{\circ} \mathrm{C}$ with $1000 \mathrm{rpm}$ mixing on a MixMate (Eppendorf, Hamburg, Germany). The next day, $4 \mu \mathrm{L}$ of each saturated culture was inoculated into a fresh $400 \mu \mathrm{L}$ of G418-containing SC -lys media and cells were grown for an additional 3 hours prior to flow cytometry. We performed all flow cytometry experiments on an LSR II flow cytometer (BD, Franklin Lakes, NJ, USA) equipped with a $20 \mathrm{~mW} 488 \mathrm{~nm}$ laser with 488/10 and 525/50 filters for measuring forward and side scatter and sfGFP fluorescence, respectively, as well as a $40 \mathrm{~mW}$ $561 \mathrm{~nm}$ laser and a 610/20 filter for measuring mCherry fluorescence. Table 4 lists the parameters and settings for all flow cytometry and fluorescence-activated cell sorting (FACS) experiments. We recorded 10,000 cells each from 8 independent biological replicates per strain for the ODC and Rpn4 degron TFTs.

\begin{tabular}{lrrl}
\hline Parameter & Laser Line $(\mathrm{nm})$ & Laser Setting $(\mathrm{V})$ & Filter \\
\hline forward scatter (FSC) & 488 & 500 & $488 / 10$ \\
side scatter (SSC) & 488 & 275 & $488 / 10$ \\
sfGFP & 488 & 500 & $525 / 50$ \\
mCherry & 561 & 615 & $610 / 20$ \\
\hline
\end{tabular}

Table 4: Flow cytometry and FACS settings.

All flow cytometry data was analyzed using $\mathrm{R}$ ( $\mathrm{R}$ Foundation for Statistical Computing, Vienna Austria) and the flowCore $\mathrm{R}$ package ${ }^{71}$. We filtered each flow cytometry dataset to exclude all events outside of $10 \% \pm$ the median forward scatter (a proxy for cell size). This gating approach captured the central peak of cells in the FSC histogram and removed cellular debris, aggregates of multiple cells, and restricted our analyses to cells of the same approximate size ${ }^{14}$. 
For the statistical analysis of flow cytometry results, we used the negative $\log _{2}$ RFP / GFP ratio as a measure of proteasome activity. We computed the negative $\log _{2}$ RFP / GFP ratio for each of 10,000 cells per strain per reporter and extracted the median from each sample for subsequent statistical analyses. We used a one-way ANOVA with Tukey's HSD post-hoc test for all between groups comparisons of proteasome activity.

\section{Fluorescence-Activated Cell Sorting}

We used FACS to collect pools of segregant cells for genetic mapping by bulk segregant analysis ${ }^{32,37}$. We thawed and inoculated segregant populations into $5 \mathrm{~mL}$ of SGA medium containing G418 and canavanine for overnight growth at $30^{\circ} \mathrm{C}$ with rolling. The following morning, we diluted $1 \mathrm{~mL}$ of cells from each segregant population into a fresh $4 \mathrm{~mL}$ of SGA medium containing G418 and canavanine. Diluted segregant cultures were grown for 4 hours prior to sorting on a FACSAria II cell sorter (BD). Plots of side scatter (SSC) height by SSC width and forward scatter (FSC) height by FSC width were used to remove doublets from each sample and cells were further filtered to contain cells within $\pm 7.5 \%$ of the central FSC peak. We empirically determined that this filtering approach excluded cellular debris and aggregates while retaining the primary haploid cell population. We also defined a fluorescence-positive population by retaining only those TFT-containing cells with sfGFP fluorescence values higher than negative control BY and RM strains without TFTs. We collected pools of 20,000 cells each from the $2 \%$ high and low proteasome activity tails (Figure 2) from two independent biological replicates (spore preparations) for each TFT. Pools of 20,000 cells were collected into sterile $1.5 \mathrm{~mL}$ polypropylene tubes containing $1 \mathrm{~mL}$ of SGA medium that were grown overnight at $30^{\circ} \mathrm{C}$ with rolling. After overnight growth, we mixed $750 \mu \mathrm{L}$ of cells with 450 $\mu \mathrm{L}$ of $40 \%(\mathrm{v} / \mathrm{v})$ glycerol and stored this mixture in $2 \mathrm{~mL} 96$ well plates at $-80^{\circ} \mathrm{C}$.

\section{Genomic DNA Isolation, Library Preparation, and Whole-Genome Sequencing}

To isolate genomic DNA from sorted segregant pools, we first pelleted $800 \mu \mathrm{L}$ of each pool by centrifugation at 3,700 rpm for 10 minutes. Supernantants were discarded and cell pellets were resuspended in $800 \mu \mathrm{L}$ of a $1 \mathrm{M}$ sorbitol solution containing $0.1 \mathrm{M}$ EDTA, $14.3 \mathrm{mM}$ $\beta$-mercaptoethanol, and $500 \mathrm{U}$ of Zymolyase lytic enzyme (United States Biological) to digest cell walls. Zymolyase digestions were carried out by resuspending cell pellets with mixing at 1000 rpm for 2 minutes followed by incubation for 2 hours at $37^{\circ} \mathrm{C}$. After completing the digestion reaction, we pelleted and resuspended cells in $50 \mu \mathrm{L}$ of phosphate buffered saline. We then used the Quick-DNA 96 Plus kit (Zymo Research, Irvine, CA, USA) to extract genomic DNA according to the manufacturer's protocol, including an overnight protein digestion in a $20 \mathrm{mg} / \mathrm{mL}$ 
proteinase $\mathrm{K}$ solution at $55^{\circ} \mathrm{C}$ prior to loading samples onto columns. DNA was eluted from sample preparation columns using $40 \mu \mathrm{L}$ of DNA elution buffer $(10 \mathrm{mM}$ Tris- $\mathrm{HCl}$ [pH 8.5], 0.1 mM EDTA). DNA concentrations for each sample were determined with the Qubit dsDNA BR assay kit (Thermo Fisher Scientific, Waltham, MA, USA) in a 96 well format using a Synergy H1 plate reader (BioTek Instruments, Winooski, VT, USA).

We used genomic DNA from our segregant pools to prepare a short-read library for whole-genome sequencing on the Illumina Next-Seq platform using a previously-described approach ${ }^{14,32,37}$. The Nextera DNA library kit (Illumina, San Diego, CA, USA) was used according to the manufacturer's instructions with the following modifications. We fragmented and added sequencing adapters to genomic DNA by adding $5 \mathrm{ng}$ of DNA to a master mix containing $4 \mu \mathrm{L}$ of Tagment DNA buffer, $1 \mu \mathrm{L}$ of sterile molecular biology grade water, and $5 \mu \mathrm{L}$ of Tagment DNA enzyme diluted 1:20 in Tagment DNA buffer and incubating this mixture on a SimpliAmp thermal cycler using the following parameters (Thermo Fisher Scientific): $55^{\circ} \mathrm{C}$ temperature, $20 \mu \mathrm{L}$ reaction volume, 10 minute incubation. We PCR amplified libraries prior to sequencing by adding $10 \mu \mathrm{L}$ of the tagmentation reaction to a master mix containing $1 \mu \mathrm{L}$ of an Illumina i5 and i7 index primer pair mixture, $0.375 \mu \mathrm{L}$ of ExTaq polymerase (Takara), $5 \mu \mathrm{L}$ of ExTaq buffer, $4 \mu \mathrm{L}$ of a dNTP mixture, and $29.625 \mu \mathrm{L}$ of sterile molecular biology grade water. To multiplex samples for sequencing, we generated all 96 possible index oligo combinations using 8 i5 and 12 i7 index primers. Libraries were PCR amplified on a SimpliAmp thermal cycler using the following parameters: initial denaturation at $95^{\circ} \mathrm{C}$ for 30 seconds, then 17 cycles of $95^{\circ} \mathrm{C}$ for 10 seconds (denaturation), $62^{\circ} \mathrm{C}$ for 30 seconds (annealing), and $72^{\circ} \mathrm{C}$ for 3 minutes (extension). The DNA concentration of each reaction was quantified using the Qubit dsDNA BR assay kit (Thermo Fisher Scientific). We pooled equimolar amounts of each sample, ran this mixture on a $2 \%$ agarose gel, and extracted and purified DNA in the $400 \mathrm{bp}$ to 600 bp region using the Monarch Gel Extraction Kit (NEB) according to the manufacturer's instructions.

The pooled library was submitted to the University of Minnesota Genomics Center (UMGC) for quality control assessment and Illumina sequencing. UMGC staff performed three quality control (QC) assays prior to sequencing. The PicoGreen dsDNA quantification reagent (Thermo Fisher Scientific) was used to determine library concentration, with a concentration $\geq 1 \mathrm{ng} / \mu \mathrm{L}$ required to pass. The Tapestation electrophoresis system (Agilent Technologies, Santa Clara, CA, USA) was used to determine library size, with libraries in the range of 200 to $700 \mathrm{bp}$ passing. Finally, the KAPA DNA Library Quantification kit (Roche, Penzberg, Germany) was used to determine library 
functionality, with libraries requiring a concentration $\geq$ than $2 \mathrm{nM}$ to pass. The submitted library passed each QC assay. The library was sequenced on a Next-Seq 550 instrument in mid-output, 75 bp paired-end mode, generating 153,887,828 reads across all samples, with a median of 9,757,090 and a range of 5,994,921 to 14,753,319 reads per sample. The mean read quality for all samples was $>30$. The median read coverage of the genome was 21 , with a range of 16 to 25 across all samples. Data will be deposited into the NIH Sequence Read Archive following publication.

\section{QTL Mapping}

We used a previously-described approach to identify QTLs from our whole-genome sequencing data $^{14,32,37}$. We initially filtered our raw reads to retain only those with a mean base quality score greater than 30 . Filtered reads were aligned to the $S$. cerevisiae reference genome (sacCer3) with the Burroughs-Wheeler alignment tool ${ }^{72}$. We used samtools ${ }^{73}$ to remove unaligned reads, non-uniquely aligned reads, and PCR duplicates and then to produce vcf files containing coverage and allelic read counts at each of 18,871 high-confidence, reliable $\mathrm{SNPs}^{36,74}$, with BY alleles as reference and RM alleles as alternative alleles.

QTLs were called from allele counts using the MULTIPOOL algorithm ${ }^{75}$. MULTIPOOL estimates a logarithm of the odds (LOD) score by calculating a likelihood ratio from two models. In the noncausal model, the locus is not associated with the trait and the high and low proteasome activity pools have the same frequency of the BY and RM alleles. In the causal model, the locus is associated with the trait, such that the BY and RM allele frequencies differ between pools. QTLs were defined as loci with a LOD $\geq$ to 4.5 . In a previous study ${ }^{14}$, we empirically determined that this threshold produces a $0.5 \%$ false discovery rate (FDR) for TFT-based genetic mapping by bulk segregant analysis. We used the following MULTIPOOL settings: bp per centiMorgan = 2,200, bin size $=100 \mathrm{bp}$, effective pool size $=1,000$. As in previous studies ${ }^{32,37}$, we excluded variants with allele frequencies higher than 0.9 or lower than $0.1^{14,32,37}$. QTL confidence intervals were defined as a 2-LOD drop from the QTL peak (the QTL position with the highest LOD value). We also computed the allele frequency difference $(\triangle \mathrm{AF})$ at each allele as a measure of each QTL's effect size and to visualize QTLs. Because allele frequencies are affected by random counting noise, we smoothed allele frequencies along the genome using loess regression prior to calculating $\Delta \mathrm{AF}$ for each sample. 


\section{Acknowledgements}

We thank the members of the Albert laboratory for critical feedback on the manuscript and the University of Minnesota's Flow Cytometry Resource and Genomics Center for their contributions to the project.

\section{Competing Interests}

The authors declare that they have no competing interests.

\section{Funding}

This work was supported by NIH grants F32-GM128302 to MAC and R35-GM124676 to FWA.

\section{Supplementary Materials}

The following supplementary materials are included with this manuscript:

Supplementary Figures 1-2

Supplementary Table 1 


\section{References}

${ }^{1}$ J. S. Bett. Proteostasis regulation by the ubiquitin system. Essays Biochem, 60(2):143-151, 10 2016 .

${ }^{2}$ J. Hanna and D. Finley. A proteasome for all occasions. FEBS Lett, 581(15):2854-2861, Jun 2007.

${ }^{3}$ C. Pohl and I. Dikic. Cellular quality control by the ubiquitin-proteasome system and autophagy. Science, 366(6467):818-822, 112019.

${ }^{4}$ D. Finley, H. D. Ulrich, T. Sommer, and P. Kaiser. The ubiquitin-proteasome system of Saccharomyces cerevisiae. Genetics, 192(2):319-360, Oct 2012. [DOI:10.1534/genetics.112.140467] [PubMed:23028185].

${ }^{5}$ G. A. Collins and A. L. Goldberg. The Logic of the 26S Proteasome. Cell, 169(5):792-806, May 2017.

${ }^{6}$ A. Varshavsky. The N-end rule pathway and regulation by proteolysis. Protein Sci., 20(8):12981345, Aug 2011.

${ }^{7}$ R. S. Marshall and R. D. Vierstra. Dynamic Regulation of the 26S Proteasome: From Synthesis to Degradation. Front Mol Biosci, 6:40, 2019.

${ }^{8}$ N. P. Dantuma and L. C. Bott. The ubiquitin-proteasome system in neurodegenerative diseases: precipitating factor, yet part of the solution. Front Mol Neurosci, 7:70, 2014.

${ }^{9}$ C. Zheng, T. Geetha, and J. R. Babu. Failure of ubiquitin proteasome system: risk for neurodegenerative diseases. Neurodegener Dis, 14(4):161-175, 2014.

${ }^{10}$ L. Petrucelli and T. M. Dawson. Mechanism of neurodegenerative disease: role of the ubiquitin proteasome system. Ann Med, 36(4):315-320, 2004.

${ }^{11}$ B. Mészáros, M. Kumar, T. J. Gibson, B. Uyar, and Z. Dosztányi. Degrons in cancer. Sci Signal, 10(470), Mar 2017.

12 A. Nencioni, F. Grunebach, F. Patrone, A. Ballestrero, and P. Brossart. The proteasome and its inhibitors in immune regulation and immune disorders. Crit Rev Immunol, 26(6):487-498, 2006.

${ }^{13}$ S. S. Wing. The UPS in diabetes and obesity. BMC Biochem, 9 Suppl 1:S6, Oct 2008. 
${ }^{14}$ Mahlon A. Collins, Gemechu Mekonnen, and Frank W. Albert. Genetic basis of variation in ubiquitin-proteasome system activity. bioRxiv, 2021.

${ }^{15}$ H. C. Yen, Q. Xu, D. M. Chou, Z. Zhao, and S. J. Elledge. Global protein stability profiling in mammalian cells. Science, 322(5903):918-923, Nov 2008.

${ }^{16}$ Y. Geffen, A. Appleboim, R. G. Gardner, N. Friedman, R. Sadeh, and T. Ravid. Mapping the Landscape of a Eukaryotic Degronome. Mol Cell, 63(6):1055-1065, 092016.

${ }^{17}$ A. Khmelinskii, P. J. Keller, A. Bartosik, M. Meurer, J. D. Barry, B. R. Mardin, A. Kaufmann, S. Trautmann, M. Wachsmuth, G. Pereira, W. Huber, E. Schiebel, and M. Knop. Tandem fluorescent protein timers for in vivo analysis of protein dynamics. Nat. Biotechnol., 30(7):708714, Jun 2012.

${ }^{18}$ J. Erales and P. Coffino. Ubiquitin-independent proteasomal degradation. Biochim Biophys Acta, 1843(1):216-221, Jan 2014.

${ }^{19}$ M. Zhang, C. M. Pickart, and P. Coffino. Determinants of proteasome recognition of ornithine decarboxylase, a ubiquitin-independent substrate. EMBO J, 22(7):1488-1496, Apr 2003.

${ }^{20}$ J. Takeuchi, H. Chen, M. A. Hoyt, and P. Coffino. Structural elements of the ubiquitinindependent proteasome degron of ornithine decarboxylase. Biochem J, 410(2):401-407, Mar 2008.

${ }^{21}$ Y. Xie and A. Varshavsky. RPN4 is a ligand, substrate, and transcriptional regulator of the 26S proteasome: a negative feedback circuit. Proc. Natl. Acad. Sci. U.S.A., 98(6):3056-3061, Mar 2001.

${ }^{22} \mathrm{~S}$. W. Ha, D. Ju, and Y. Xie. The N-terminal domain of Rpn4 serves as a portable ubiquitinindependent degron and is recognized by specific 19S RP subunits. Biochem Biophys Res Commun, 419(2):226-231, Mar 2012.

${ }^{23}$ D. Ju and Y. Xie. Proteasomal degradation of RPN4 via two distinct mechanisms, ubiquitindependent and -independent. J Biol Chem, 279(23):23851-23854, Jun 2004.

${ }^{24}$ M. A. Hoyt, M. Zhang, and P. Coffino. Ubiquitin-independent mechanisms of mouse ornithine decarboxylase degradation are conserved between mammalian and fungal cells. J Biol Chem, 278(14):12135-12143, Apr 2003. 
${ }^{25}$ G. Mannhaupt, R. Schnall, V. Karpov, I. Vetter, and H. Feldmann. Rpn4p acts as a transcription factor by binding to PACE, a nonamer box found upstream of $26 \mathrm{~S}$ proteasomal and other genes in yeast. FEBS Lett, 450(1-2):27-34, Apr 1999.

${ }^{26}$ A. V. Morozov, D. S. Spasskaya, D. S. Karpov, and V. L. Karpov. The central domain of yeast transcription factor Rpn4 facilitates degradation of reporter protein in human cells. FEBS Lett, 588(20):3713-3719, Oct 2014.

${ }^{27}$ M. A. Hoyt, M. Zhang, and P. Coffino. Probing the ubiquitin/proteasome system with ornithine decarboxylase, a ubiquitin-independent substrate. Methods Enzymol, 398:399-413, 2005.

${ }^{28}$ I. Momose, D. Tatsuda, S. Ohba, T. Masuda, D. Ikeda, and A. Nomoto. In vivo imaging of proteasome inhibition using a proteasome-sensitive fluorescent reporter. Cancer Sci, 103(9):17301736, Sep 2012.

${ }^{29}$ F. Duveau, W. Toubiana, and P. J. Wittkopp. Fitness Effects of Cis-Regulatory Variants in the Saccharomyces cerevisiae TDH3 Promoter. Mol Biol Evol, 34(11):2908-2912, Nov 2017.

${ }^{30}$ B. P. Metzger, D. C. Yuan, J. D. Gruber, F. Duveau, and P. J. Wittkopp. Selection on noise constrains variation in a eukaryotic promoter. Nature, 521(7552):344-347, May 2015.

${ }^{31}$ F. W. Albert, J. S. Bloom, J. Siegel, L. Day, and L. Kruglyak. Genetics of trans-regulatory variation in gene expression. Elife, 7, 072018.

${ }^{32}$ F. W. Albert, S. Treusch, A. H. Shockley, J. S. Bloom, and L. Kruglyak. Genetics of single-cell protein abundance variation in large yeast populations. Nature, 506(7489):494-497, Feb 2014.

33 A. Khmelinskii and M. Knop. Analysis of protein dynamics with tandem fluorescent protein timers. Methods Mol. Biol., 1174:195-210, 2014.

${ }^{34}$ A. Khmelinskii, M. Meurer, C. T. Ho, B. Besenbeck, J. F?ller, M. K. Lemberg, B. Bukau, A. Mogk, and M. Knop. Incomplete proteasomal degradation of green fluorescent proteins in the context of tandem fluorescent protein timers. Mol. Biol. Cell, 27(2):360-370, Jan 2016.

${ }^{35} \mathrm{D}$. Ju, H. Xu, X. Wang, and Y. Xie. The transcription activation domain of Rpn4 is separate from its degrons. Int J Biochem Cell Biol, 42(2):282-286, Feb 2010.

${ }^{36}$ I. M. Ehrenreich, N. Torabi, Y. Jia, J. Kent, S. Martis, J. A. Shapiro, D. Gresham, A. A. Caudy, and L. Kruglyak. Dissection of genetically complex traits with extremely large pools of yeast segregants. Nature, 464(7291):1039-1042, Apr 2010. 
${ }^{37}$ C. Brion, S. M. Lutz, and F. W. Albert. Simultaneous quantification of mRNA and protein in single cells reveals post-transcriptional effects of genetic variation. Elife, 9, Nov 2020.

${ }^{38}$ R. W. Michelmore, I. Paran, and R. V. Kesseli. Identification of markers linked to diseaseresistance genes by bulked segregant analysis: a rapid method to detect markers in specific genomic regions by using segregating populations. Proc Natl Acad Sci U S A, 88(21):9828-9832, Nov 1991.

${ }^{39}$ M. Gaisne, A. M. Bécam, J. Verdière, and C. J. Herbert. A 'natural' mutation in Saccharomyces cerevisiae strains derived from S288c affects the complex regulatory gene HAP1 (CYP1). Curr Genet, 36(4):195-200, Oct 1999.

${ }^{40}$ I. M. Ehrenreich, J. P. Gerke, and L. Kruglyak. Genetic dissection of complex traits in yeast: insights from studies of gene expression and other phenotypes in the BYxRM cross. Cold Spring Harb Symp Quant Biol, 74:145-153, 2009.

${ }^{41}$ R. B. Wickner. MKT1, a nonessential Saccharomyces cerevisiae gene with a temperaturedependent effect on replication of M2 double-stranded RNA. J Bacteriol, 169(11):4941-4945, Nov 1987.

${ }^{42}$ T. F. Mackay, E. A. Stone, and J. F. Ayroles. The genetics of quantitative traits: challenges and prospects. Nat Rev Genet, 10(8):565-577, Aug 2009.

${ }^{43}$ R. B. Brem, G. Yvert, R. Clinton, and L. Kruglyak. Genetic dissection of transcriptional regulation in budding yeast. Science, 296(5568):752-755, Apr 2002.

${ }^{44}$ M. Zhang, A. I. MacDonald, M. A. Hoyt, and P. Coffino. Proteasomes begin ornithine decarboxylase digestion at the C terminus. J Biol Chem, 279(20):20959-20965, May 2004.

${ }^{45}$ P. Fort, A. V. Kajava, F. Delsuc, and O. Coux. Evolution of proteasome regulators in eukaryotes. Genome Biol Evol, 7(5):1363-1379, May 2015.

${ }^{46}$ C. Gille, A. Goede, C. Schlöetelburg, R. Preissner, P. M. Kloetzel, U. B. Göbel, and C. Frömmel. A comprehensive view on proteasomal sequences: implications for the evolution of the proteasome. J Mol Biol, 326(5):1437-1448, Mar 2003.

${ }^{47}$ D. Komander and M. Rape. The ubiquitin code. Annu Rev Biochem, 81:203-229, 2012.

${ }^{48}$ F. Ohtake, Y. Saeki, S. Ishido, J. Kanno, and K. Tanaka. The K48-K63 Branched Ubiquitin Chain Regulates NF-B Signaling. Mol Cell, 64(2):251-266, 102016. 
${ }^{49}$ M. E. French, C. F. Koehler, and T. Hunter. Emerging functions of branched ubiquitin chains. Cell Discov, 7(1):6, Jan 2021.

${ }^{50}$ X. M. Li, D. Y. Chao, Y. Wu, X. Huang, K. Chen, L. G. Cui, L. Su, W. W. Ye, H. Chen, H. C. Chen, N. Q. Dong, T. Guo, M. Shi, Q. Feng, P. Zhang, B. Han, J. X. Shan, J. P. Gao, and H. X. Lin. Natural alleles of a proteasome 2 subunit gene contribute to thermotolerance and adaptation of African rice. Nat Genet, 47(7):827-833, Jul 2015.

${ }^{51}$ G. M. Rosewarne, R. P. Singh, J. Huerta-Espino, H. M. William, S. Bouchet, S. Cloutier, H. McFadden, and E. S. Lagudah. Leaf tip necrosis, molecular markers and beta1-proteasome subunits associated with the slow rusting resistance genes Lr46/Yr29. Theor Appl Genet, 112(3):500-508, Feb 2006.

${ }^{52}$ K. E. Mason, J. K. Hilmer, W. S. Maaty, B. D. Reeves, P. A. Grieco, B. Bothner, and A. M. Fischer. Proteomic comparison of near-isogenic barley (Hordeum vulgare L.) germplasm differing in the allelic state of a major senescence QTL identifies numerous proteins involved in plant pathogen defense. Plant Physiol Biochem, 109:114-127, Dec 2016.

${ }^{53}$ H. Wang, M. Jiang, H. Zhu, Q. Chen, P. Gong, J. Lin, J. Lu, and J. Qiu. Quantitative assessment of the influence of PSMA6 variant (rs1048990) on coronary artery disease risk. Mol Biol Rep, 40(2):1035-1041, Feb 2013.

54 M. G. Heckman, A. I. Soto-Ortolaza, N. N. Diehl, S. Rayaprolu, T. G. Brott, Z. K. Wszolek, J. F. Meschia, and O. A. Ross. Genetic variants associated with myocardial infarction in the PSMA6 gene and Chr9p21 are also associated with ischaemic stroke. Eur $J$ Neurol, 20(2):300-308, Feb 2013.

55 J. de Ligt, M. H. Willemsen, B. W. van Bon, T. Kleefstra, H. G. Yntema, T. Kroes, A. T. Vultovan Silfhout, D. A. Koolen, P. de Vries, C. Gilissen, M. del Rosario, A. Hoischen, H. Scheffer, B. B. de Vries, H. G. Brunner, J. A. Veltman, and L. E. Vissers. Diagnostic exome sequencing in persons with severe intellectual disability. N Engl J Med, 367(20):1921-1929, Nov 2012.

${ }^{56}$ A. Kröll-Hermi, F. Ebstein, C. Stoetzel, V. Geoffroy, E. Schaefer, S. Scheidecker, S. Bär, M. Takamiya, K. Kawakami, B. A. Zieba, F. Studer, V. Pelletier, C. Eyermann, C. Speeg-Schatz, V. Laugel, D. Lipsker, F. Sandron, S. McGinn, A. Boland, J. F. Deleuze, L. Kuhn, J. Chicher, P. Hammann, S. Friant, C. Etard, E. Krüger, J. Muller, U. Strähle, and H. Dollfus. Proteasome subunit PSMC3 variants cause neurosensory syndrome combining deafness and cataract due to proteotoxic stress. EMBO Mol Med, 12(7):e11861, 072020. 
${ }^{57}$ S. Küry, T. Besnard, F. Ebstein, T. N. Khan, T. Gambin, J. Douglas, C. A. Bacino, W. J. Craigen, S. J. Sanders, A. Lehmann, X. Latypova, K. Khan, M. Pacault, S. Sacharow, K. Glaser, E. Bieth, L. Perrin-Sabourin, M. L. Jacquemont, M. T. Cho, E. Roeder, A. S. Denommé-Pichon, K. G. Monaghan, B. Yuan, F. Xia, S. Simon, D. Bonneau, P. Parent, B. Gilbert-Dussardier, S. Odent, A. Toutain, L. Pasquier, D. Barbouth, C. A. Shaw, A. Patel, J. L. Smith, W. Bi, S. Schmitt, W. Deb, M. Nizon, S. Mercier, M. Vincent, C. Rooryck, V. Malan, I. Briceño, A. Gómez, K. M. Nugent, J. B. Gibson, B. Cogné, J. R. Lupski, H. A. F. Stessman, E. E. Eichler, K. Retterer, Y. Yang, R. Redon, N. Katsanis, J. A. Rosenfeld, P. M. Kloetzel, C. Golzio, S. Bézieau, P. Stankiewicz, and B. Isidor. De Novo Disruption of the Proteasome Regulatory Subunit PSMD12 Causes a Syndromic Neurodevelopmental Disorder. Am J Hum Genet, 100(2):352363, Feb 2017.

58 J. M. Heward, A. Allahabadia, M. C. Sheppard, A. H. Barnett, J. A. Franklyn, and S. C. Gough. Association of the large multifunctional proteasome (LMP2) gene with Graves' disease is a result of linkage disequilibrium with the HLA haplotype DRB1*0304-DQB1*02-DQA1*0501. Clin Endocrinol (Oxf), 51(1):115-118, Jul 1999.

${ }^{59}$ A. V. Gomes. Genetics of proteasome diseases. Scientifica (Cairo), 2013:637629, 2013.

60 Jana M. Mitchell, James Nemesh, Sulagna Ghosh, Robert E. Handsaker, Curtis J. Mello, Daniel Meyer, Kavya Raghunathan, Heather de Rivera, Matt Tegtmeyer, Derek Hawes, Anna Neumann, Ralda Nehme, Kevin Eggan, and Steven A. McCarroll. Mapping genetic effects on cellular phenotypes with "cell villages". bioRxiv, 2020.

${ }^{61}$ A. Khmelinskii, E. Blaszczak, M. Pantazopoulou, B. Fischer, D. J. Omnus, G. Le Dez, A. Brossard, A. Gunnarsson, J. D. Barry, M. Meurer, D. Kirrmaier, C. Boone, W. Huber, G. Rabut, P. O. Ljungdahl, and M. Knop. Protein quality control at the inner nuclear membrane. Nature, 516(7531):410-413, Dec 2014.

62 J. D. Pedelacq, S. Cabantous, T. Tran, T. C. Terwilliger, and G. S. Waldo. Engineering and characterization of a superfolder green fluorescent protein. Nat. Biotechnol., 24(1):79-88, Jan 2006.

${ }^{63}$ N. C. Shaner, R. E. Campbell, P. A. Steinbach, B. N. Giepmans, A. E. Palmer, and R. Y. Tsien. Improved monomeric red, orange and yellow fluorescent proteins derived from Discosoma sp. red fluorescent protein. Nat. Biotechnol., 22(12):1567-1572, Dec 2004. 
${ }^{64}$ S. Prakash, L. Tian, K. S. Ratliff, R. E. Lehotzky, and A. Matouschek. An unstructured initiation site is required for efficient proteasome-mediated degradation. Nat Struct Mol Biol, 11(9):830837, Sep 2004.

${ }^{65}$ A. L. Goldstein and J. H. McCusker. Three new dominant drug resistance cassettes for gene disruption in Saccharomyces cerevisiae. Yeast, 15(14):1541-1553, Oct 1999.

${ }^{66}$ J. H. Zwolshen and J. K. Bhattacharjee. Genetic and biochemical properties of thialysine-resistant mutants of Saccharomyces cerevisiae. J Gen Microbiol, 122(2):281-287, Feb 1981.

${ }^{67}$ A. Baryshnikova, M. Costanzo, S. Dixon, F. J. Vizeacoumar, C. L. Myers, B. Andrews, and C. Boone. Synthetic genetic array (SGA) analysis in Saccharomyces cerevisiae and Schizosaccharomyces pombe. Methods Enzymol, 470:145-179, 2010.

${ }^{68}$ E. Kuzmin, M. Costanzo, B. Andrews, and C. Boone. Synthetic Genetic Array Analysis. Cold Spring Harb Protoc, 2016(4):pdb.prot088807, Apr 2016.

${ }^{69}$ R. D. Gietz and R. H. Schiestl. High-efficiency yeast transformation using the LiAc/SS carrier DNA/PEG method. Nat Protoc, 2(1):31-34, 2007.

${ }^{70}$ A. C. Ward. Rapid analysis of yeast transformants using colony-PCR. Biotechniques, 13(3):350, Sep 1992.

${ }^{71}$ F. Hahne, N. LeMeur, R. R. Brinkman, B. Ellis, P. Haaland, D. Sarkar, J. Spidlen, E. Strain, and R. Gentleman. flowCore: a Bioconductor package for high throughput flow cytometry. $B M C$ Bioinformatics, 10:106, Apr 2009.

${ }^{72}$ H. Li and R. Durbin. Fast and accurate short read alignment with Burrows-Wheeler transform. Bioinformatics, 25(14):1754-1760, Jul 2009.

${ }^{73}$ H. Li, B. Handsaker, A. Wysoker, T. Fennell, J. Ruan, N. Homer, G. Marth, G. Abecasis, and R. Durbin. The Sequence Alignment/Map format and SAMtools. Bioinformatics, 25(16):20782079, Aug 2009.

${ }^{74}$ J. S. Bloom, I. M. Ehrenreich, W. T. Loo, T. L. Lite, and L. Kruglyak. Finding the sources of missing heritability in a yeast cross. Nature, 494(7436):234-237, Feb 2013.

${ }^{75}$ M. D. Edwards and D. K. Gifford. High-resolution genetic mapping with pooled sequencing. BMC Bioinformatics, 13 Suppl 6:S8, Apr 2012. 


\section{Supplementary Materials}

\section{ODC TFT}
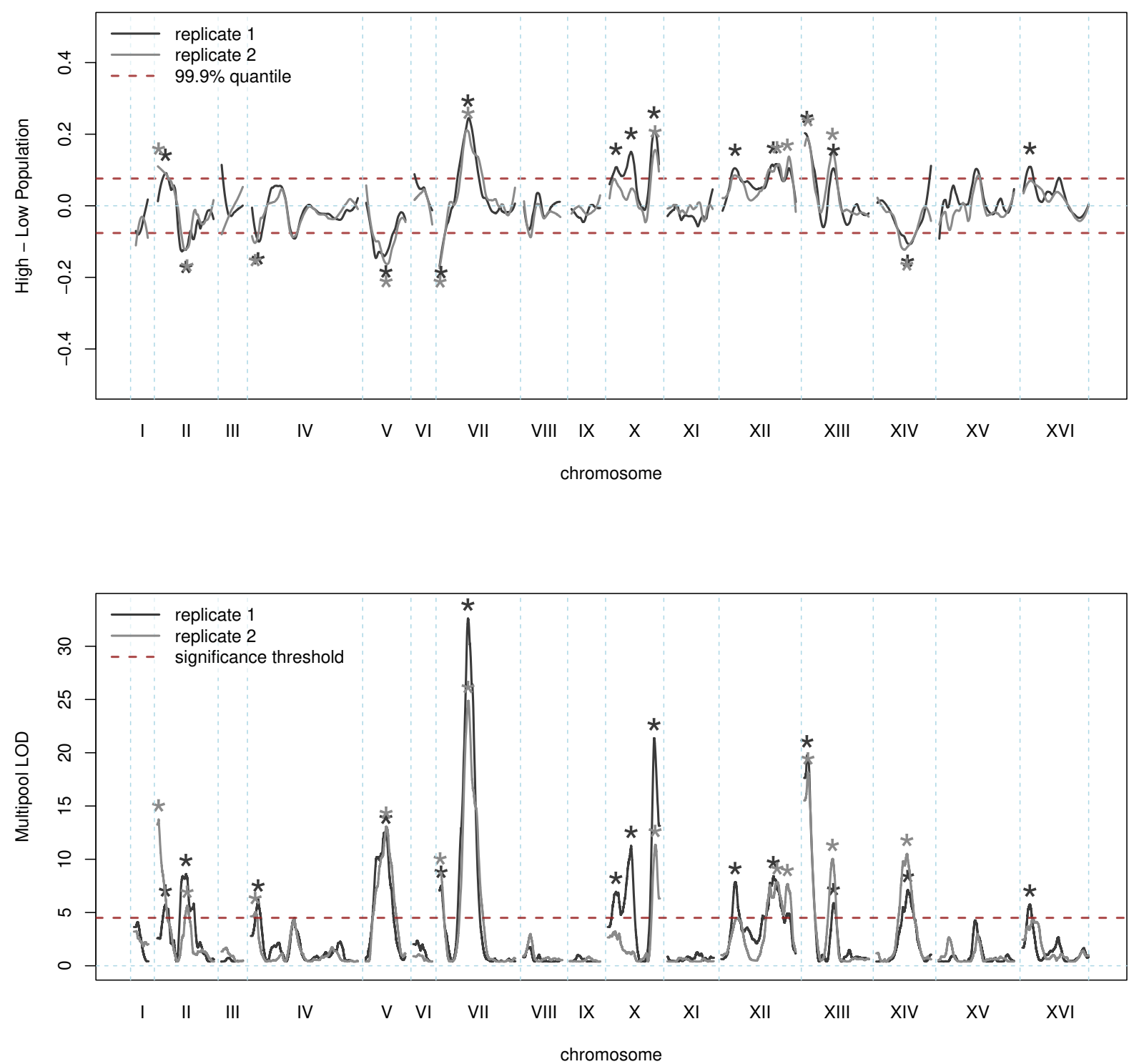

Supplementary Figure 1. ODC TFT QTL mapping results. Top. The loess smoothed RM allele frequency difference (high proteasome activity pool minus low proteasome activity pool) is plotted across the S. cerevisiae genome. The dashed red lines mark the empirically determined 99.9\% quantile. Bottom. LOD scores for ODC TFT QTL mapping. The dashed red line indicates the empirically determined LOD score significance threshold (4.5), which results in a $0.5 \%$ FDR. For both panels, asterisks denote QTLs, colored by independent biological replicate. 

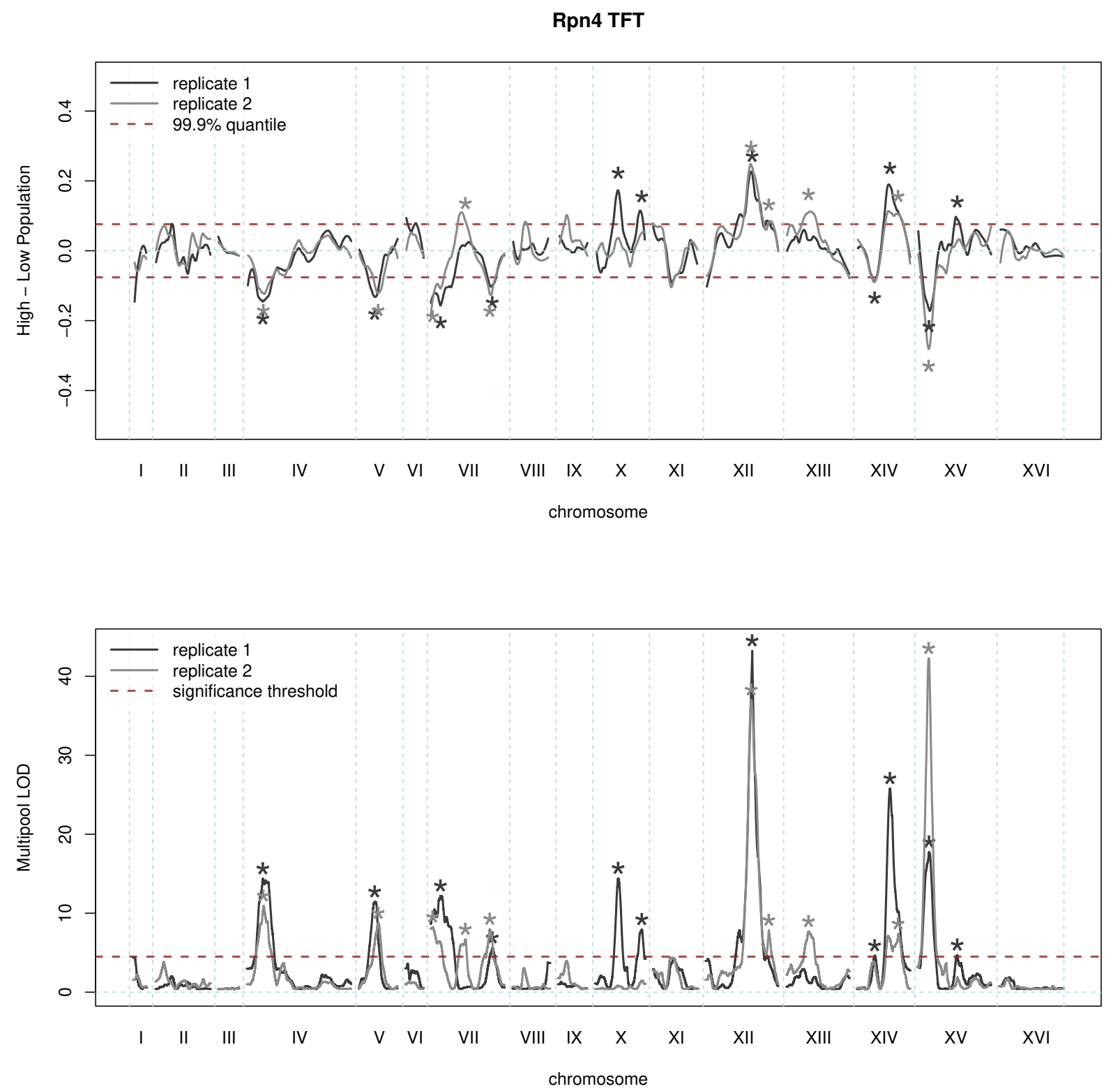

Supplementary Figure 2. Rpn 4 TFT QTL mapping results. Top. The loess smoothed RM allele frequency difference (high proteasome activity pool minus low proteasome activity pool) is plotted across the S. cerevisiae genome. The dashed red lines mark the empirically determined 99.9\% quantile. Bottom. LOD scores for Rpn 4 TFT QTL mapping. The dashed red line indicates the empirically determined LOD score significance threshold (4.5), which results in a $0.5 \%$ FDR. For both panels, asterisks denote QTLs, colored by independent biological replicate. 
710

\begin{tabular}{lcccccc}
\hline Reporters & Chromosome & LOD & AFD & Peak Position & Left Index & Right Index \\
\hline ODC TFT/N-end Rule (3) & IVa & 7.12 & -0.108 & 78825 & 5600 & 110075 \\
ODC TFT/Rpn4 TFT/N-end Rule (8) & VIIa & 9.24 & -0.149 & 125200 & 91475 & 166375 \\
ODC TFT/N-end Rule (10) & VIIb & 16.21 & 0.173 & 409450 & 389250 & 436750 \\
Rpn4 TFT/N-end Rule (3) & VIIc & 7.42 & -0.115 & 873575 & 839950 & 903725 \\
ODC TFT/N-end Rule (3) & X & 11.19 & 0.141 & 626100 & 588400 & 660250 \\
ODC TFT/Rpn4 TFT/N-end Rule (5) & XII & 10.21 & 0.113 & 665600 & 636800 & 690250 \\
ODC TFT/N-end Rule (2) & XIIIa & 16.95 & 0.170 & 47800 & 25200 & 75850 \\
ODC TFT/N-end Rule (1) & XIIIb & 15.345 & 0.1573 & 341525 & 366875 & 394975 \\
ODC TFT/N-end Rule (9) & XIVa & 32.50 & -0.226 & 464275 & 448675 & 478825 \\
Rpn4 TFT/N-end Rule (8) & XV & 18.30 & -0.176 & 163350 & 138600 & 186200 \\
\hline
\end{tabular}

Supplementary Table 1. Overlap of proteasome activity QTLs with UPS N-end rule QTLs. Numbers in parentheses in the "Reporters" column indicate how many of 20 possible UPS N-end Rule reporters the corresponding proteasome activity QTL was detected for. Numeric columns display the median value calculated from the set of proteasome activity and UPS N-end Rule reporters for which the QTL was detected. "Peak Position", "Left Index", and "Right Index" refer to base pair positions on the indicated chromosome. 